\title{
Heat transfer in a ribbed square duct by Large-Eddy-Simulation
}

\author{
O. Labbé \\ ONERA, Châtillon, France
}

\begin{abstract}
Large-eddy simulation is used to understand the flow in a square ribbed duct representative of systems designed for internal cooling of turbine blades. The presence of ribs increases turbulence levels and enhances heat transfer. The objective of the present LES study is to solve three-dimensional Navier-Stokes equations for a compressible flow to enable simulations for which the temperature variation within the flow is very significant. This simulation allows us to deal with a fully developed turbulent flow and heat transfer in a ribbed channel with a high blockage ratio, whose parameters are chosen to reproduce the experiments of Casarsa et al. "Characterization of the velocity and heat transfer fields in an internal cooling channel with high blockage ratio." Proceedings of ASME TURBO EXPO 2002 June 3-6, Amsterdam, The Netherlands, 2002. The simulation is restricted to one pitch length and periodic conditions are applied in the streamwise direction. Mean and turbulent quantities are presented, together with the heat transfer.

Keywords: LES, turbulence, heat transfer, rib, compressible flow.
\end{abstract}

\section{Introduction}

The efficient design of the internal ducts in gas turbine blades requires a detailed knowledge of the flow and heat phenomena occurring inside these passages. In ducts, ribs are used to disturb the boundary layers, thereby promoting turbulence and enhancing heat transfer. The presence of the ribs leads to a complex velocity field with regions of flow separation upstream and downstream of the ribs and contributes to the level of mixing of the cooler part of the air stream with the warmer air close to the walls.

A large number of experimental investigations are available in the literature on aerodynamics and heat transfer performance of internal cooling channels with 
ribs. Among them one may cite Casarsa et al. [1], who dealt with a detailed aero/thermal investigation of the turbulent flow in an internal cooling channel with high blockage ratio.

A large amount of numerical studies by DNS or LES investigations has been devoted to the analysis of the flow and heat transfer in a ribbed channel. The flow is affected by the sidewall and a three-dimensional representation is needed. Among the studies, which consider 3D flow, one may list: Murata and Mochizuki [2], Watanabe and Takahashi [3], Tafti [4], the large-eddy simulations carried out by Lohász et al. [5-7] reproduce the experiments of Casarsa et al. [1] without taking into account the heat transfer. All of the papers mentioned above solve the Navier-Stokes equations for an incompressible flow and a temperature equation which acts like a passive scalar.

The objective of the present LES study is to solve three-dimensional NavierStokes equations for a compressible flow to enable simulations for which the temperature variation within the flow is very significant. These simulations allow us to deal with a fully developed turbulent flow and heat transfer in a ribbed channel with high blockage ratio, whose parameters are chosen to reproduce the experiments of Casarsa et al. [1].

\section{Periodic square ribbed channel}

The present contribution concerns the simulation of the flow in a square duct where successive ribs of square cross section are mounted on the lower wall perpendicularly to the stream direction. Casarsa et al. [1] have experimentally found that for such a configuration the flow starts to repeat itself in every pitch length after the fourth rib. This situation allows us to confine the numerical simulation to investigate around a single rib and to use periodic conditions in the streamwise $(\mathrm{X})$ direction. The numerical results will be compared with those obtained experimentally between the fourth and fifth ribs. The channel is characterized by a square cross section $(\mathrm{Y}, \mathrm{Z}) 0.1 \mathrm{x} 0.1 \mathrm{~m}^{2}$. The rib cross section (hxh) is $0.03 \times 0.03 \mathrm{~m}^{2}$, providing a blockage ratio of $30 \%$. The pitch-to-height ratio $(\mathrm{P} / \mathrm{h})$ is equal to 10 . The Reynolds number of the mean flow based on the hydraulic diameter $D_{h}$ and the bulk velocity is equal to $4.10^{4}$. The duct walls as well as the three faces of the rib exposed to the main flow are heated by imposing a constant temperature $\mathrm{T}_{\mathrm{w}}$ in order to represent the constant heat flux $\mathrm{q}_{\mathrm{w}}$ prescribed in the experiments. Fig. 1 shows a schematic of the channel with a transverse rib located on the bottom wall of the duct.

\section{Governing equations and computational model}

The time-accurate resolution method of complex flows, which present separating /reattaching shear layers and secondary flows is based on the large-eddy simulation. LES, by solving the energy containing eddies, reduces the complexity of Direct Numerical Simulation (DNS) by several orders of magnitude. Two approaches are commonly used to perform LES: explicit subgrid stress models are used to represent the effect of unresolved scales of 
motion on the large scales and the second method consists in using the Monotonic Integrated Large-Eddy Simulations (MILES) proposed by Boris et al. [8]. This latter is based on the assumption that the intrinsic dissipation of an upwind scheme is able to mimic the dissipative behaviour of the unresolved turbulent scales, and that when using such a scheme, the subgrid viscosity has to be set to zero. The MILES approach has been used in the present study. The governing equations for LES are the grid-filtered mass, momentum and total energy equations for compressible flow. In order to simplify the resulting set of equations, a mass-weighted change of variable (Favre) is defined as: $\quad \overline{\rho f}=\bar{\rho} \widetilde{f}$

$$
\begin{aligned}
& \frac{\partial \bar{\rho}}{\partial t}+\frac{\partial}{\partial x_{j}}\left(\bar{\rho} \tilde{u}_{j}\right)=0 \\
& \frac{\partial}{\partial t}\left(\bar{\rho} \tilde{u}_{i}\right)+\frac{\partial}{\partial x_{j}}\left(\bar{\rho} \tilde{u}_{i} \tilde{u}_{j}\right)+\frac{\partial \bar{p}}{\partial x_{i}}-\frac{\partial \widetilde{\sigma}_{i j}}{\partial x_{j}}=-\alpha \delta_{i 1} \\
& \frac{\partial \hat{\rho E}}{\partial t}+\frac{\partial}{\partial x_{j}}\left\{(\hat{\rho} E+\bar{p}) \tilde{u}_{j}\right\}-\frac{\partial}{\partial x_{j}}\left(\widetilde{\sigma}_{i j} \tilde{u}_{i}\right)+\frac{\partial \widetilde{q}_{j}}{\partial x_{j}}=-\alpha \tilde{u}_{1}-\bar{\rho} \tilde{u}_{1} \beta
\end{aligned}
$$

where the total energy is written as follows:

$$
\hat{\rho} E=\frac{\bar{p}}{\gamma-1}+\frac{1}{2} \bar{\rho} \tilde{u}_{j} \tilde{u}_{j}
$$

The stress tensor $\widetilde{\sigma}_{i j}$ is defined as:

$$
\widetilde{\sigma}_{i j}=\mu(\widetilde{T})\left(\frac{\partial \tilde{u}_{i}}{\partial x_{j}}+\frac{\partial \tilde{u}_{j}}{\partial x_{i}}-\frac{2}{3} \frac{\partial \tilde{u}_{k}}{\partial x_{k}} \delta_{i j}\right) .
$$

The heat flux vector $\widetilde{q}_{j}$ is given by:

$$
\widetilde{q}_{j}=-\frac{\mu(\widetilde{T})}{(\gamma-1) \operatorname{Re} \operatorname{Pr} M^{2}} \frac{\partial \widetilde{T}}{\partial x_{j}} .
$$

Due to the periodicity of the simulation the term $\alpha$ is the mean pressure gradient to balance the wall-shear stress on the walls, it is imposed in the streamwise direction to maintain a constant flow rate and $\delta_{i 1}$ takes the value of one when $i=1$. A mean enthalpy gradient $\beta$ is added in order to balance the global energy due to the heated walls, $\beta=q_{w} S_{w} / \dot{m} P$ where $\mathrm{q}_{\mathrm{w}}$ is the heat flux at the walls, $\mathrm{S}_{\mathrm{w}}$ the total surface of the heated walls, $\dot{m}$ the flow rate and $\mathrm{P}$ is the pitch distance. To close the set of equations, the following filtered equation of state is used: $\tilde{p} / \bar{\rho}=r \widetilde{T}$ with $r=287 \mathrm{~J} \mathrm{~kg}^{-1} \mathrm{~K}^{-1}$. The viscosity dependence on temperature is modelled using the Sutherland's law. 


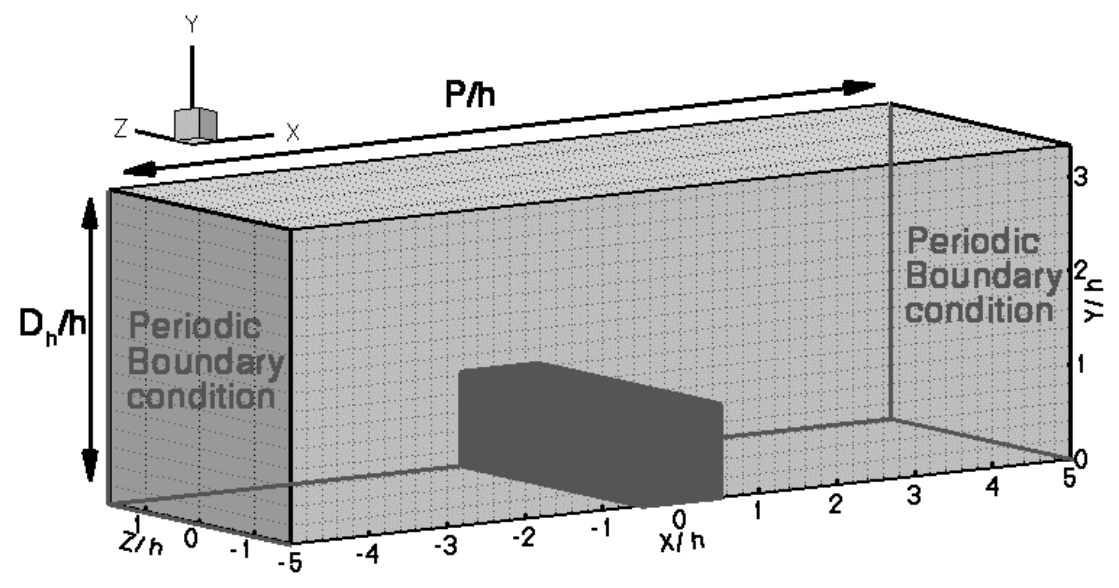

Figure 1: Computational domain of the square ribbed channel.

\section{Numerical schemes and boundary conditions}

The governing equations for mass, momentum and energy are discretised with a conservative finite-volume formulation. The spatial discretisation method is based on the cell-centred finite volume methodology. An upwind biased, with a third-order MUSCL interpolation scheme of AUSM+(P) family without any shock capturing feature is used for the convective scheme. An explicit time integration is carried out by means of a third-order compact Runge-Kutta scheme. Details of the method can be found in Péchier et al. [9] and in Larchevêque et al. [10]. Periodic boundary conditions are imposed in the streamwise $(\mathrm{X})$ direction. No-slip boundary conditions and wall temperature are applied at the top, bottom and lateral walls of the channel and at the top and lateral walls of the rib.

\section{Simulation characteristics}

Mesh density is relatively high in the vicinity of the rib and the duct surfaces to resolve the boundary /shear layers, which is crucial to the accurate prediction of turbulence and heat transfer. The computational domain consists in about 1.15 million grid points, which are distributed as follows: 201 and 61 grid points respectively in the streamwise and spanwise directions. In the vertical direction 101 (61 above the rib) grid points are used. The size of the first cell is $0.003 \mathrm{D}_{\mathrm{h}}$ near walls. In wall units the distance from the wall of the first point is $\mathrm{y}^{+}=5$ based a posteriori on the mean wall shear velocity. The time step is set to $10^{-5} \mathrm{D}_{\mathrm{h}} / \mathrm{U}_{0}$ corresponding to an average CFL value of 0.1 over the whole domain. The initialization of the field is achieved assuming an initial bulk velocity corresponding to the experiments and the calculations are integrated on time 
until the flow rate adjusts to balance between internal losses and the specified pressure gradient. Once the flow has reached a stationary state (30 time units), sampling to obtain mean and turbulent quantities is carried out for 120 time units. All the results are normalized by the bulk flow velocity $\left(\mathrm{U}_{0}\right)$ in order to compare them with previous experimental and/or numerical works.

\section{Results and discussion}

The flow is strongly three-dimensional, but in the centre plane $\mathrm{Z} / \mathrm{h}=0$, the flow is essentially two-dimensional. The presence of the high spanwise velocity regions upstream of the rib is due to the blockage effect of the rib. As a matter of fact the blockage forces the flow in front of the rib to swirl toward the lateral walls in an upward motion over the rib which is noticeable in Fig. 2. In this latter, the streamlines started from a line upstream from the rib, are spiralling in front of it, forming a surface trapped in the upstream corner. This forces the flow to swirl towards the walls above the rib and to finally escape downstream in the channel corner.

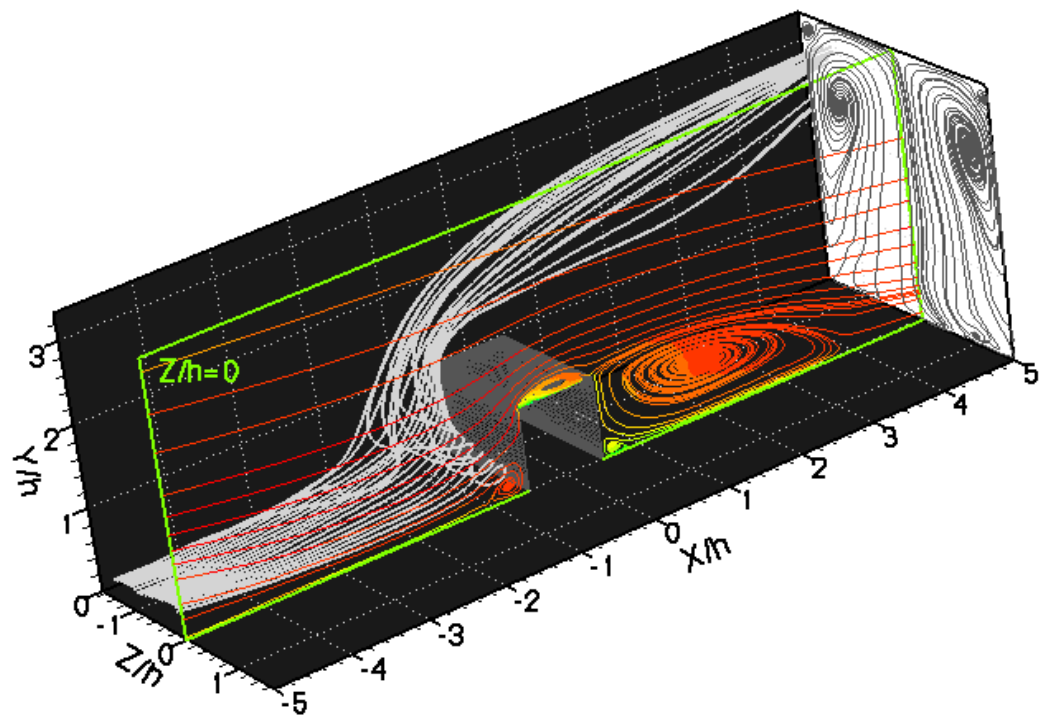

Figure 2: Global view of the flow.

Considering the mean flow in the centre plane $\mathrm{Z} / \mathrm{h}=0$., streamlines of Fig. 3 reveal four distinct zones associated with the ribs. A wide recirculation bubble occurs downstream from the rib due to the sudden expansion of the flow, accompanied by a small counter rotating vortex V1 at the lower corner behind the rib. After the flow reattachment line, a new boundary layer develops and impinges on the next rib forming an upstream vortex V3. Another recirculation zone V2 is formed on the rib top as the sharp front edge of the rib deflects the 
flow. The sizes of the different recirculation zones are compared with measurements of Casarsa et al. [1] and with the LES results of Lohász et al. [6] and summarized in Table 1. The comparison shows a good agreement with experimental measurements except for the vortex V1, which is larger for both LES simulations.

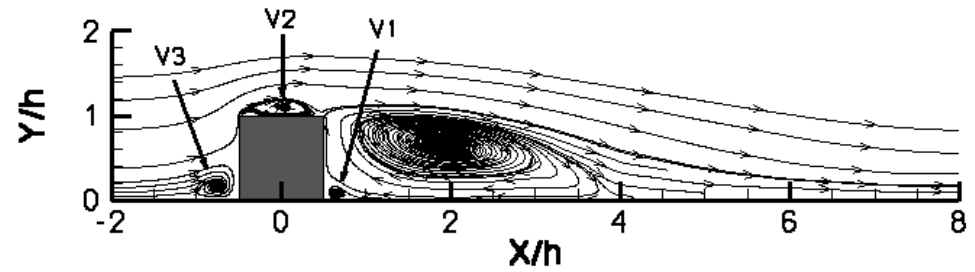

Figure 3: Flow structures in $\mathrm{Z} / \mathrm{h}=0$.

Table 1: Comparison of the different recirculation zones.

\begin{tabular}{|c|c|c|c|c|}
\hline & Reattachment & V1 & V2 & V3 \\
\hline Casarsa et al. [1] & $4.26<\mathrm{X} / \mathrm{h}<4.34$ & $\begin{array}{ll}0.255 & <\Delta \mathrm{X} / \mathrm{h} \\
<0.28 & \end{array}$ & $0.6<\Delta \mathrm{X} / \mathrm{h}<0.9$ & $1.04<\Delta \mathrm{X} / \mathrm{h}<1.5$ \\
\hline $\begin{array}{l}\text { LES (Lohász et } \\
\text { al.) [6] }\end{array}$ & $\Delta \mathrm{X} / \mathrm{h}=3.85$ & $\Delta \mathrm{X} / \mathrm{h}=0.5$ & $\Delta \mathrm{X} / \mathrm{h}=0.91$ & $\Delta \mathrm{X} / \mathrm{h}=0.66$ \\
\hline Present LES & $\Delta \mathrm{X} / \mathrm{h}=4.4$ & $\Delta \mathrm{X} / \mathrm{h}=0.6$ & $\Delta \mathrm{X} / \mathrm{h}=0.7$ & $\Delta \mathrm{X} / \mathrm{h}=1.3$ \\
\hline
\end{tabular}
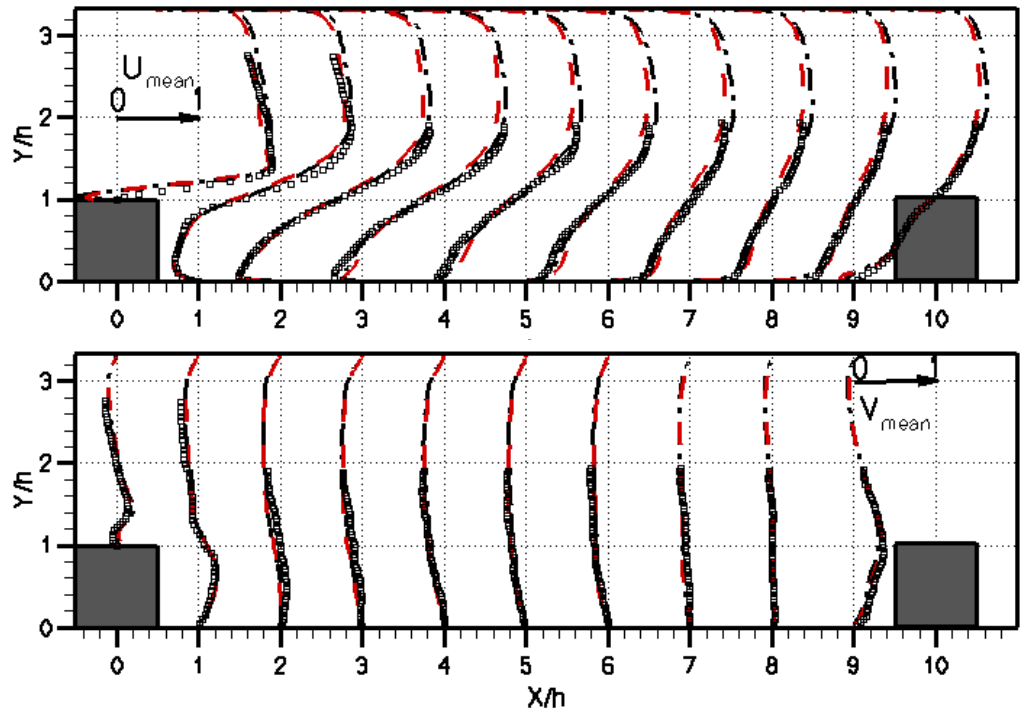

Figure 4: Mean streamwise (top) and normal (bottom) velocity profiles at $\mathrm{Z} / \mathrm{h}=0$. 
The mean streamwise and normal velocity profiles are compared in Fig. 4 on the centre plane $\mathrm{Z} / \mathrm{h}=0$. The LES of Lohász et al. [7] in a red dashed line. The experimental results plotted with black squares and the present simulation in black dotted/dashed line show a very good agreement. The rib reduces the cross section and consequently accelerates the flow, and high velocity gradients are found in the upstream top corner of the rib. The sudden expansion downstream from it leads to a large recirculation region associated with a strong shear layer.

All the planes shifted from the wall $(0.05)$ in Fig. 5 are coloured by mean streamwise velocity. On three transverse sections: $\mathrm{X} / \mathrm{h}=0$ (on the $\mathrm{rib}$ ), $\mathrm{X} / \mathrm{h}=4$ (reattachment line) and $\mathrm{X} / \mathrm{h}=9.45$ (in front of the rib), surface streamlines are plotted and allow us to understand the flow complexity. Above the rib, the flow is characterized by an acceleration of the fluid and the streamline patterns clearly indicate how it moves towards the lateral walls. Counter-rotating structures compensate the downward current. Two other counter-rotating structures appear and develop in the upper corners of the transverse sections. Moreover volume streamrods are plotted to follow the flow through the computational domain, one of them passes over the rib and then is trapped in the main recirculation region, another spirals on the top of the rib toward the lateral wall.

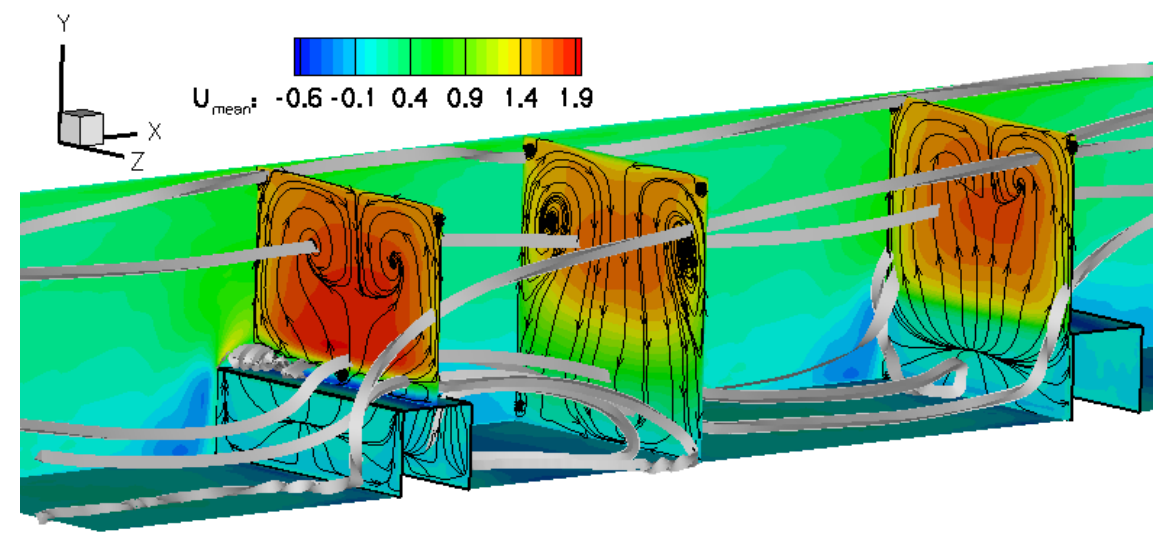

Figure 5: Secondary flow patterns and volume streamrods.

Figure 6 shows contours of resolved $U_{r m s}, V_{r m s}$ (normalized by $U_{0}$ ) and resolved turbulent shear stresses U'V' (normalized by $\mathrm{U}_{0}{ }^{2}$ ) at the centre plane $\mathrm{Z} / \mathrm{h}=0$. The LES results (right) are in good agreement with those obtained experimentally by Casarsa et al. [1] (left). The streamwise fluctuations $\mathrm{U}_{\mathrm{rms}}$ reach their maximum value in the separated shear layer at the leading edge of the rib. They are lowest above the rib and in the recirculation region immediately behind the rib. The vertical fluctuations $\mathrm{V}_{\mathrm{rms}}$ reach their maximum in front of the rib as well as in the separated shear layer downstream from the rib and they are low behind the rib in the stagnation region. The maximum of turbulent shear stress U'V' occurs in a very small region at the leading edge of the rib in the separated shear layer. 
444 Computational Methods and Experimental Measurements XIII

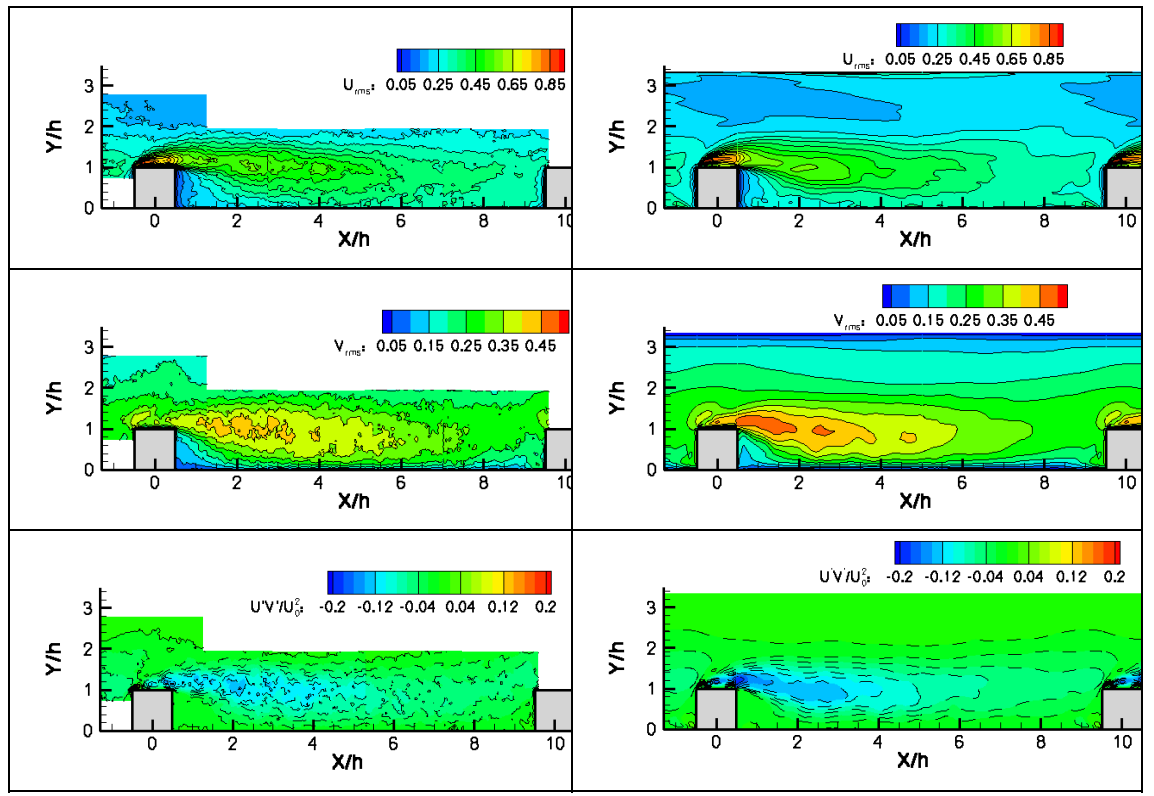

Figure 6: $\mathrm{U}_{\text {rms }}, \mathrm{V}_{\text {rms }}$ and $\mathrm{U}^{\prime} \mathrm{V}^{\prime}$ in $\mathrm{Z} / \mathrm{h}=0$, present LES (right), Casarsa et al. (left).

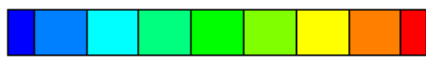

$\mathrm{Nu} / \mathrm{Nu}_{0}, \quad 0.5 \quad 0.70 .91 .11 .31 .51 .71 .9$

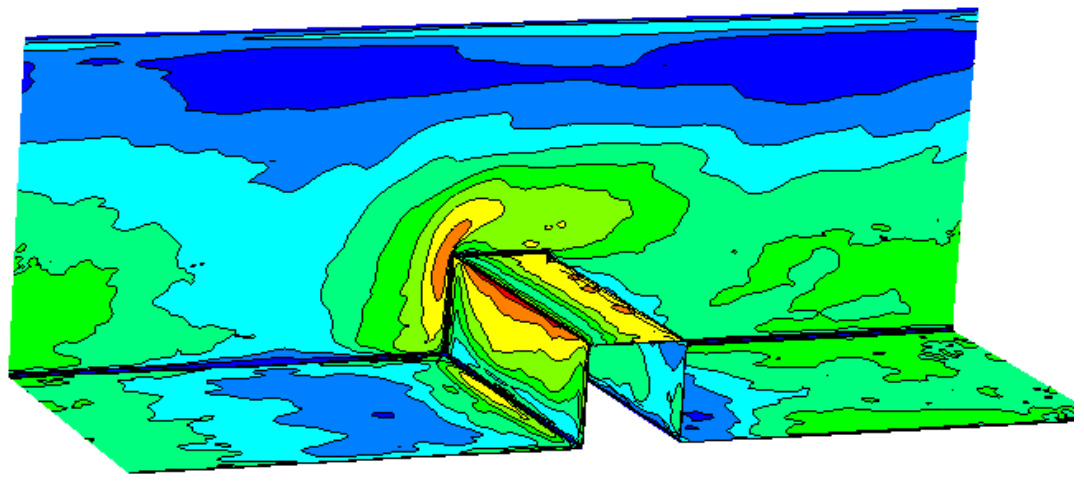

Figure 7: Distribution of Nusselt number on ribbed wall, lateral wall and rib.

The heat transfer results are presented in terms of an enhancement factor which corresponds to the ratio between Nusselt number $\mathrm{Nu}$ of the flow at the wall $\left(N u=q_{w} D_{h} / \lambda\left(\theta_{w}-\theta_{r e f}\right)\right)$ and $\mathrm{Nu}_{0}$ obtained from Dittus-Boelter correlation: $\mathrm{Nu}_{0}=0.023 \mathrm{Re}^{0.8} \mathrm{Pr}^{0.4}$. The results are plotted on Fig. 7 on the 
different walls. On the rib itself, the heat transfer is maximum at the top leading edge as a result of the strong fluid acceleration, and in the reattachment region of the structure V2. On the lateral wall, the highest values of $\mathrm{Nu} / \mathrm{Nu}_{0}$ are found in a region arched over the top front corner of the rib, due to the upward motion described previously with streamlines in Fig. 5. In front of the rib on the ribbed wall, the heat transfer is high as the result of highly unsteady secondary eddies produced in this region. However, it drops on moving closer to the lateral walls. Immediately downstream from the rib, the heat transfer is minimum. Further downstream in the main recirculation region the Nusselt number increases, but does not reach the peaks previously described. Compared to the experimental results of Casarsa et al. [1], the predicted heat transfer is found at the same places, but is underestimated. In the LES simulation a mean enthalpy gradient is added due to the periodic condition in the streamwise direction, but this term does not accurately balance the energy increase due to the heated walls, and globally the temperature is increasing in the flow and the heat transfer is reducing at the walls. A wall temperature is a priori imposed in order to assume the heat flux imposed in the experiments, but as the whole flow heats, the correct heat flux is not obtained a posteriori.

\section{Conclusion}

LES has been performed in a ribbed square duct with a rib height to hydraulic diameter ratio of .3 and a rib pitch to rib height ratio of 10 for a bulk Reynolds number of 40,000. The simulation reproduces the major flow structures measured experimentally with a very good agreement, namely a recirculation zone formed on the top of the rib and behind the rib, the lateral impingement of the flow on the side walls. After the flow reattachment, the boundary layer grows up and impinges on the rib forming another vortex. The three-dimensional nature of the flow is very evident. It is observed that the rib induces a flow swirling towards the sidewalls before passing away on top of the rib. A comparison of mean flow with experiments by Casarsa and LES of Lohász shows excellent agreement. Moreover the contour plots turbulence intensity in the centre plane are in good agreement with those obtained experimentally by Casarsa.

The sidewalls have a strong influence on the heat transfer distribution. The streamlines connecting bottom wall upstream the rib and a region over the top corner of the rib are well correlated to the high values of heat transfer. While the heat transfer on the ribbed wall is enhanced due to the streamwise flow, the secondary flow plays a major role in the heat transfer on the side walls. Immediately behind the rib, a secondary recirculation is trapped between the wall and the primary recirculation zone, which prevents the fluid from efficiently mixing with the fluid in the channel core, but increases the heat transfer on the downstream rib side. The heat transfer locations of LES results compare well with experimental results, however their levels are under-predicted, due to an unsuitable enthalpy gradient, inefficient to maintain an imposed heat flux at the walls. 


\section{References}

[1] Casarsa, L. Çakan, M. \& Arts, T., Characterization of the velocity and heat transfer fields in an internal cooling channel with high blockage ratio. Proceedings of ASME TURBO EXPO 2002 June 3-6, Amsterdam, The Netherlands, 2002.

[2] Murata, A. \& Mochizuki, S., Large eddy simulation with a dynamic subgrid-scale model of turbulent heat transfer in an orthogonally rotating rectangular duct with transverse rib turbulators, Int. J. Heat and Mass Transfer 43, pp. 1243-1259, 2000.

[3] Watanabe, K. \& Takahashi, T., LES simulation and experimental measurement of fully developed ribbed channel flow and heat transfer, Proceedings of ASME TURBO EXPO June 3-6, Amsterdam, The Netherlands, 2002.

[4] Tafti, D.K., Evaluating the role of subgrid stress modelling in a ribbed duct for the internal cooling of turbine blades, Int. J. Heat and Fluid Flow 26, pp. 92-104, 2005.

[5] Lohász, M.M., Rambaud, P. \& Benocci, C., LES simulation of ribbed square duct flow with FLUENT and comparison with PIV data, Conference on Modelling Fluid Flow (CMFF'03), The $12^{\text {th }}$ International Conference on Fluid Flow Technologies, Budapest, Hungary, September 3-6, 2003.

[6] Lohász, M.M., Rambaud, P. \& Benocci, C., MILES flow inside a square section ribbed duct, RTO Meeting, AVT-120 Workshop on " Urban Dispersion Modelling” April 1-2, Rhode Saint Genèse, Belgium, 2004.

[7] Lohász, M.M., Rambaud, P. \& Benocci, C., Flow features in a fully developed ribbed duct flow as a result of LES, Proceedings of the ERCOFTAC International Symposium on Engineering Turbulence Modelling and Measurements ETMM6 Sardinia, Italy, 23-25 May, 2005.

[8] Boris, J.P., Grinstein, F.F., Oran, E.S. \& Kolbe, R.L., New insights into large eddy simulation. Fluid dynamics research, 10, pp. 199-228, 1992.

[9] Péchier, M., Guillen, Ph. \& Cayzac, R., Magnus effect over finned projectiles, J. of Spacecraft and Rockets, 38(4), pp. 542-549, 2001.

[10] Larchevêque, L., Sagaut, P., Mary, I., Labbé, O. \& Comte, P., Large-eddy simulation of a compressible flow past a deep cavity. Phys. of Fluids 15(1), pp. 193-210, 2003. 